\title{
Anatomy of Rouviere's Sulcus and Its Association with Complication of Laparoscopic Cholecystectomy
}

\author{
Abhijeet Kumar $\mathbb{D}^{1},{ }^{1}$ Rupesh Shah, ${ }^{1}$ Narendra Pandit $\mathbb{D},{ }^{2}$ Suresh Prasad Sah, ${ }^{1}$ \\ and Rakesh Kumar Gupta ${ }^{1}$ \\ ${ }^{1}$ MIS Division, Department of Surgery, BPKIHS, Dharan, Nepal \\ ${ }^{2}$ Surgical Gastroenterology Division, Department of Surgery, BPKIHS, Dharan, Nepal \\ Correspondence should be addressed to Abhijeet Kumar; abhijeetkr639@gmail.com
}

Received 7 May 2020; Accepted 10 July 2020; Published 24 August 2020

Academic Editor: Peng Hui Wang

Copyright (c) 2020 Abhijeet Kumar et al. This is an open access article distributed under the Creative Commons Attribution License, which permits unrestricted use, distribution, and reproduction in any medium, provided the original work is properly cited.

\begin{abstract}
Background and Objective(s). Rouviere's sulcus (RS) is an important anatomical landmark. The aim of this study was to find the distribution of anatomical characteristics of RS seen during laparoscopic cholecystectomy in the population of our part of the world and its association with complications. Methods. This is a prospective observational study involving patients of age $\geq 16$ years who underwent laparoscopic cholecystectomy for uncomplicated gall stone at BPKIHS between May and July 2019. Result. 230 cases were analyzed, and RS was present in $90.4 \%$. Open sulcus type was the commonest (54\%), followed by scar type (22.9\%), closed sulcus type (12.5\%), and slit type (10.6\%), respectively. In $59.1 \%$ of cases, it was oblique to the anterior, inferior, and external edge of the liver, while in the remaining cases, it was transverse. The mean \pm SD values for operative time and duration of hospital stay in the RS visible and the RS not visible groups were $29.16 \pm 8.736$ and $42.9 \pm 23.646$ minutes, and $1.26 \pm 0.440$ and $1.90 \pm 0.910$ days, respectively ( $p$ value $\leq 0.001$ ). One minor complication occurred in each group: RS initially visible group and RS visible on the adhesion release group, while 3 minor complications occurred in the RS not visible group. Only one major complication occurred in the RS not visible group. Conclusion. Identification of RS by operating surgeons is a predictor of safe laparoscopic cholecystectomy.
\end{abstract}

\section{Introduction}

The Rouviere's sulcus (RS) represents a cleft, anterior to the traditionally described "segment 1 of liver" which has been recently described by Couinaud as segment IX [1]. Whatever is previously known about the sulcus came to us from some seminal studies on liver anatomy by Reynaud, Gans, and Couinaud who just noted that this sulcus was present in the majority [1-3].

Hugh et al. were the first to draw attention to its importance during laparoscopic cholecystectomy because it accurately indicates the plane of common bile duct (CBD) [4]. However, if one considers the anatomy of the RS, there are only limited studies in the literature. Its frequency and morphology are not well defined. This study was undertaken to describe the detail anatomy of RS in the population of our part of the world and to discuss the critical aspects of incorporating this useful landmark in safe cholecystectomy.

We believe that this study may increase awareness regarding variations in anatomy of Rouviere's sulcus and guide surgeons to perform safe laparoscopic cholecystectomy.

\section{Aim}

We aim to find the distribution of anatomical characteristics of RS seen during laparoscopic cholecystectomy in the population of our part of the world and its association with bile duct injury and other comorbidities.

\section{Objectives}

3.1. Primary Objective. Our primary objective is to measure the distribution of RS (present/absent) and its type (open/ 
close/slit/scar; oblique/transverse) in the population of our part of the world.

3.2. Secondary Objective. Our secondary objective is to find the association of RS with incidence of complications (such as bile duct injury, port site infection/hematoma, and others, occurring within 2 weeks of surgery) and other morbidities such as operative time, conversion to open procedure, and duration of hospital stay.

\section{Materials and Methods}

This is a prospective observational study involving humans, with single institution-based setting and purposive sampling technique. The study period was 3 months (from May to July 2019). All patients of age $\geq 16$ years with uncomplicated gall stone disease who underwent laparoscopic cholecystectomy at BPKIHS were included in the study. The exclusion criteria included the following: age $<16$ years, complicated gall stone disease, patients willing to undergo open cholecystectomy, and being not fit for general anesthesia

4.1. Sample Size Calculation. Few studies on similar topics are available in literature. In a study by Dahmane et al., open type Rouviere's sulcus (RS) was found in 70\% of livers [5]. Therefore, we calculated the sample size using Cochran's formula (at 95\% CI and 90\% power) as follows:

$$
N=\frac{Z^{2} p q}{L^{2}},
$$

where $Z$-value $=1.96$ at $95 \%$ confidence, $p=70, q=30$, and $L=10 \%$ of $p=7$.

By adding 20\% to the sample size for nonresponse error, a total of 198 patients would have been adequate for the study.

However, during the study period, we encountered 230 patients fulfilling the inclusion criteria, and all were enrolled in the study.

4.2. Ethical Clearance. The study was performed in accordance with the principle of the Declaration of Helsinki and was approved by the Institutional Review Board on 26 March 2019.

\subsection{Conceptual Framework}

4.3.1. Enrollment of Patients. All patients of age $\geq 16$ years with uncomplicated gall stone disease who underwent laparoscopic cholecystectomy at BPKIHS from May to July 2019 were enrolled in the study. A detailed clinical history was recorded in a preset proforma. Ultrasonography of abdomen and pelvis and other relevant investigations as per protocol were performed. All patients were screened for exclusion criteria, and if any was present, they were excluded. After informed consent, they were posted for elective laparoscopic cholecystectomy.
Following general anesthesia, port placement, positioning, and proper gallbladder traction, RS whether initially visible to the operating surgeon or not was noted. If RS was not initially visible, the likely reason (such as omental/bowel adhesion, absence of RS, cirrhosis of liver) was noted, and, in case of adhesion, on release of adhesion whether RS became visible before dividing the cystic artery/duct or not was noted. In RS visible group (either initially or following adhesion release), RS characteristics (in terms of its type: open sulcus/closed sulcus/slit/scar; orientation: oblique/horizontal) were noted by the operating surgeon. The dimensions (width and depth) of the RS were measured intracorporeally by comparison with the standard distance between tips of two jaws of dissecting forceps. If the sulcus was deep and wide enough (centimetric), it was described as deep sulcus. Further, a note was made as to whether the deep sulcus was open or closed, depending on whether its medial end was open towards the porta hepatis making the portal structures visible or closed towards the porta hepatis. If the sulcus was too small in its depth and breadth (subcentimetric) so that only length was measurable, it was called slit sulcus. In case of invisible RS even on release of adhesion, the likely reason (such as absence of RS, cirrhosis of liver) was noted. In all cases, complications (recognized intraoperatively or within 2 weeks postoperatively) if any, operative time, and duration of hospital stay were noted. The data was then analyzed.

4.4. Operational Definitions. The sulcus was categorized anatomically as either deep sulcus, narrow (slit), or scar. If the sulcus was deep and wide enough (centimetric), it was described as deep sulcus. Further, a note was made as to whether the deep sulcus was open (Figure 1) or closed (Figure 2), depending on whether its medial end was open towards the porta hepatis making the portal structures visible or closed towards the porta hepatis.

If the sulcus was too small in its depth and breadth (subcentimetric) so that only length was measurable, it was called slit sulcus (Figure 3 ).

Sometimes the sulcus might have just appeared as a scar only and it may be even absent (Figure 4).

We have considered bile duct injury (identified intraoperatively or postoperatively) as major complication, while other complications such as port site hematoma, port site SSI, and serous/serosanguinous collection in GB fossa were considered as minor complications.

4.5. Statistical Analysis. Data was entered in Microsoft Excel and analyzed by SPSS 11. Data was analyzed using descriptive statistics for RS characteristics and patient data, chi square or Fisher exact test to compare categorical data, and Student's $t$ test (if data is normally distributed) or Mann-Whitney $U$ test (if data is not normally distributed) for continuous data. A $p$ value of less than 0.05 was considered statistically significant throughout. 


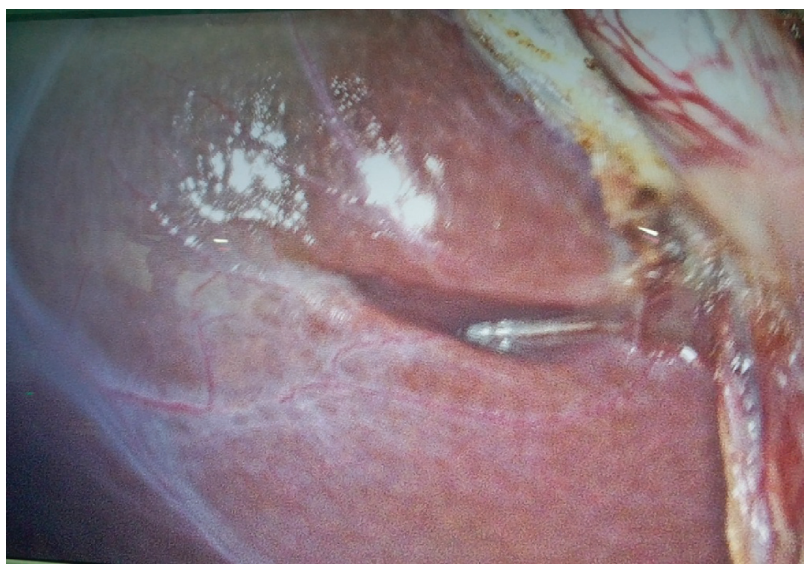

Figure 1: Open type, transverse RS.

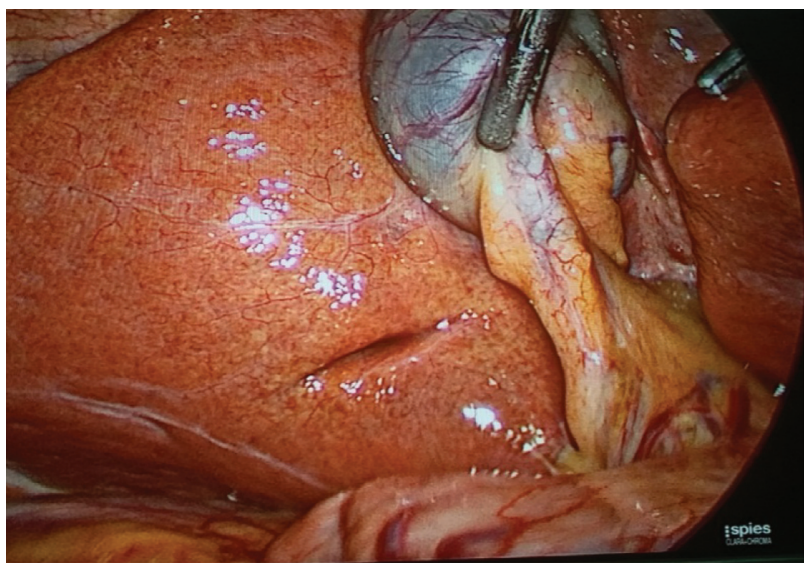

FIgUre 2: Closed type, oblique RS.

\section{Results}

A total of 233 patients were enrolled in the study. 3 patients were excluded from the study. Figure 5 illustrates overall findings of the study.

5.1. Age Distribution. The age of the patients enrolled in the study ranged from 16 to 80 years with a mean age of $41.56 \pm 14.27$ years. The majority were in the range of $26-35$ years of age (Figure 6).

5.2. Gender Distribution. In the study, there were 67 (29.1\%) males and $163(70.9 \%)$ females with female preponderance of gall stone with ratio of 2.43:1 (Figure 7).

\subsection{Visibility of RS following Port Placement, Positioning, and} Proper Traction of Gallbladder. Following port placement, positioning, and proper traction of gallbladder, RS was initially visible to the operating surgeons in 162 (70\%) cases, and in $68(30 \%)$ cases, RS was not initially visible. The reasons for initial invisibility of RS were omental adhesion in $48(70.6 \%)$ cases, absence of RS in $16(23.5 \%)$ cases, and omentum-bowel adhesion in 4 (5.9\%) cases. Out of 52 cases

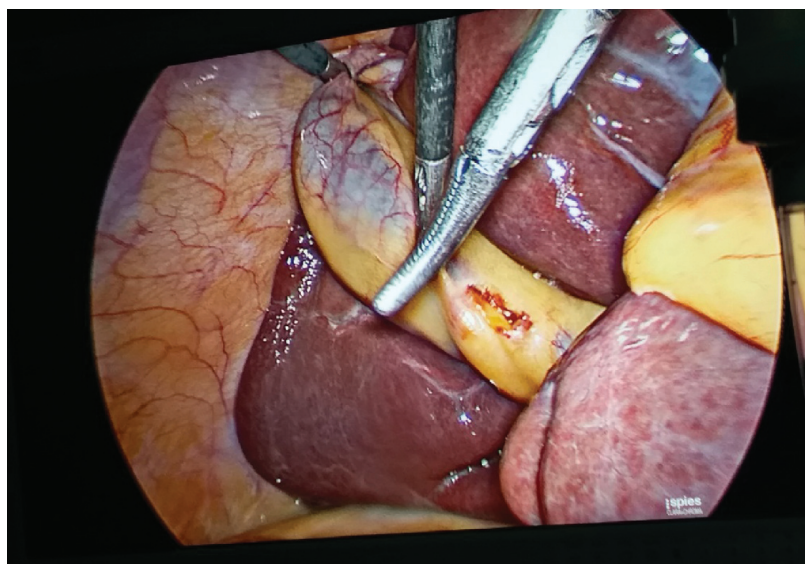

FIGURE 3: Slit type, oblique RS.

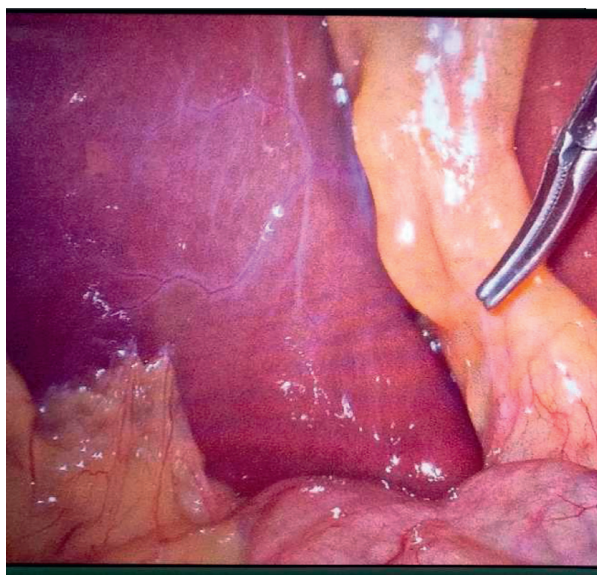

Figure 4: Absence of RS.

of initially invisible RS due to adhesion, on release of adhesion in 46 cases, RS became visible to the operating surgeon before dividing cystic artery/duct, while in 6 cases, RS was not visible because of its absence.

5.4. RS Characteristics. RS was present in 208 (90.4\%) cases, of which open sulcus type, closed sulcus type, slit type, and scar type RS were identified in 114 (54.0\%), 26 (12.5\%), 22 (10.6\%), and $46(22.9 \%)$ cases, respectively.

In $123(59.1 \%)$ cases, it was oblique to the anterior, inferior, and external edge of the liver, and in $85(40.9 \%)$ cases, it was transverse.

5.5. Operative Time and RS. The mean $\pm \mathrm{SD}$ values for operative time in the RS visible and the RS not visible groups were $29.16 \pm 8.736$ and $42.9 \pm 23.646$ minutes, respectively $(p$ value $\leq 0.001$ ) (Table 1 ).

5.6. Duration of Hospital Stay and RS. The mean \pm SD values for duration of hospital stay in the RS visible and the RS not visible groups were $1.26 \pm 0.440$ and $1.90 \pm 0.910$ days, respectively ( $p$ value $\leq 0.001)$ (Table 1$)$. 


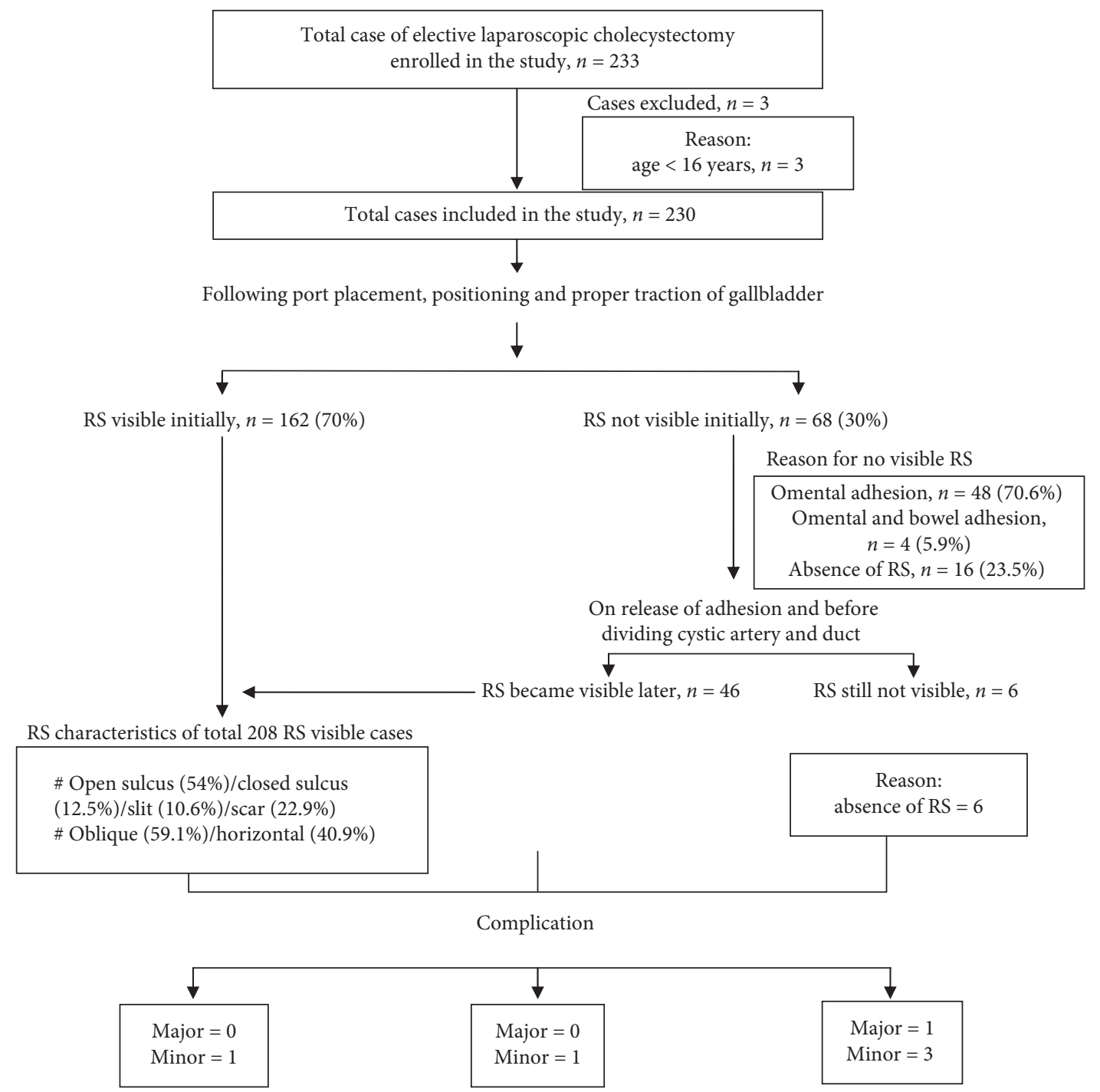

FIgURE 5: Overall findings of the study.

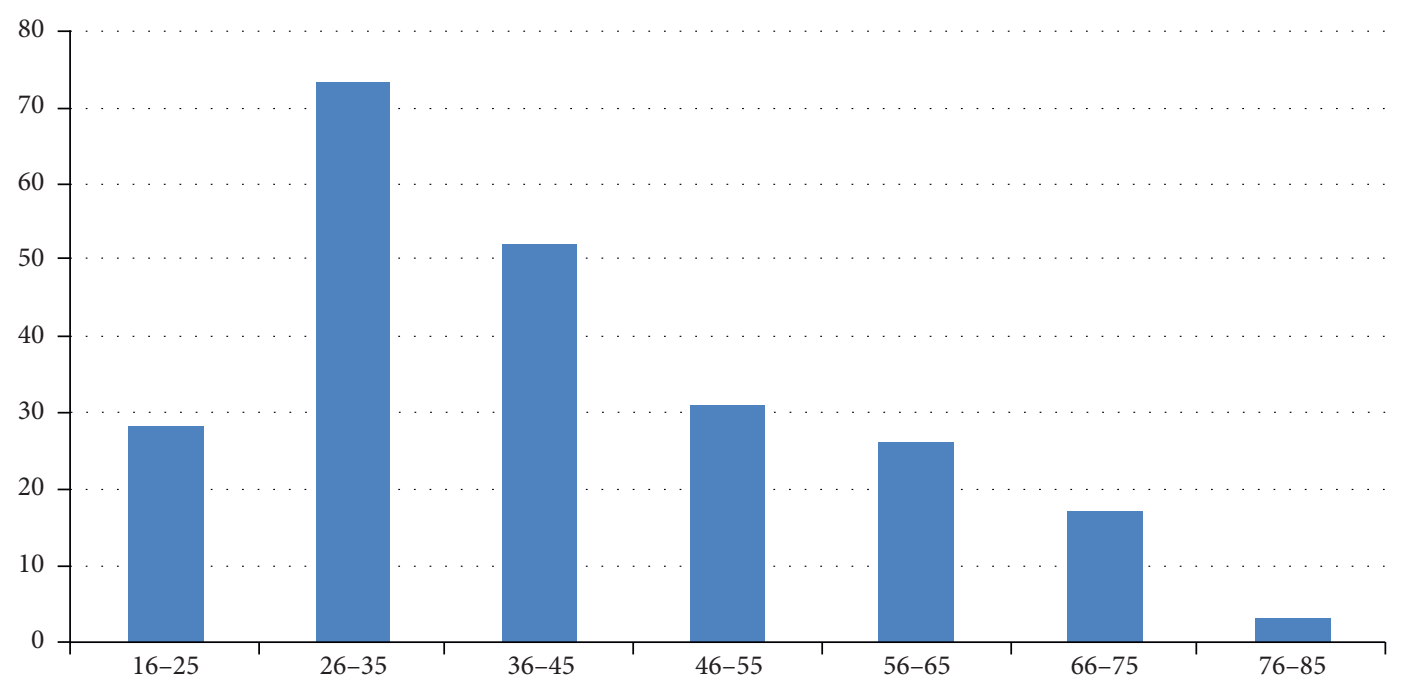

Figure 6: Age distribution. 


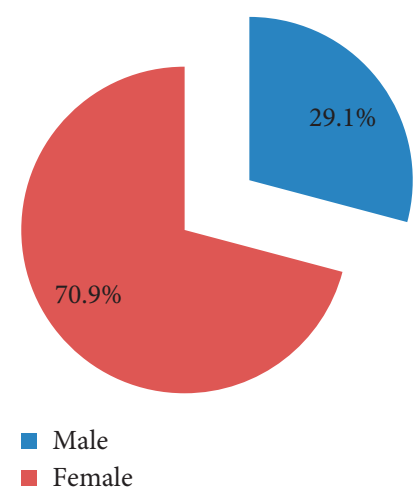

Figure 7: Gender distribution.

TABLE 1: Correlation of operative time and duration of hospital stay with RS.

\begin{tabular}{|c|c|c|c|c|}
\hline & RS visible & RS not visible & $t$-test & $p$ value \\
\hline Operative time (minute), mean $\pm S D$ & $29.16 \pm 8.736$ & $42.9 \pm 23.646$ & 6.432 & $<0.001$ \\
\hline Duration of hospital stay (day), mean \pm SD & $1.26 \pm 0.440$ & $1.90 \pm 0.910$ & 4.86 & $<0.001$ \\
\hline
\end{tabular}

5.7. Intra/Postoperative Complications and RS. Only 2 cases in RS visible before dividing cystic artery/duct group developed complications (port site hematoma: 1; serosanguineous collection in GB fossa: 1) as shown in Table 2. Port site hematoma was managed by evacuation, and serosanguineous collection in GB fossa was managed by image guided aspiration under antibiotic coverage.

4 cases in RS not visible before dividing cystic artery/duct group developed complications (port site hematoma: 1; port site SSI: 1; serosanguineous collection in GB fossa: 1; bile duct injury: 1 ) as shown in Table 2 . Port site hematoma was managed by evacuation, port site SSI was managed by dressing and antibiotic coverage as per culture sensitivity, and serosanguineous collection in GB fossa was managed by image guided aspiration under antibiotic coverage. The one in which common bile duct (CBD) injury occurred was managed by conversion to open procedure by the experienced surgeon, and hepaticojejunostomy was performed.

Hence, incidence of bile duct injury (BDI) in our study was 1 in 230 cases $(0.43 \%)$.

\section{Discussion}

This Rouviere's sulcus is seen very clearly during laparoscopic cholecystectomy due to the pressure of $\mathrm{CO}_{2}$ insufflation opening up the sulcus widely and due to the enhanced illumination and image quality of the laparoscopic camera.

6.1. Age Distribution. The mean age of patients in our study was $41.56 \pm 14.27$ years, suggesting that uncomplicated gall stone is a disease of middle age population. This is in accordance with the study by Sachdeva et al. in which the mean age was $41.5 \pm 15.4$ years [6].

The majority in our study were in the range of 26-35 years of age. This suggests a shift in the trend of gall stone disease from middle to young age, and this is in accordance with the study by Gupta et al. [7].

6.2. Gender Distribution. In our study, we observed that females had slightly higher preponderance of gall stone with female to male ratio of $2.43: 1$. In a study by Sachdeva et al., female had higher preponderance of gall stone with female to male ratio of $1.56: 1$ [6].

The sex hormones are believed to place females at higher risk. Estrogen increases biliary cholesterol secretion causing supersaturation of bile.

6.3. RS Characteristics. RS was present in around $90 \%$ of the patients, and the commonest one was the open sulcus type followed by scar type, closed sulcus type, and slit type RS, respectively. Dahmane et al. reported that Rouviere's sulcus was present in $82 \%$ of normal livers, and open RS was the commonest [5]. Reynaud et al. and Hugh et al. noted RS in $73 \%$ and $78 \%$ of livers, respectively $[2,4]$.

In our study, RS was oblique to the anterior, inferior, and external edge of the liver in $123(59.1 \%)$ cases, while in 85 $(40.9 \%)$ cases, it was transverse. In the study by Dahmane et al., the sulcus was oblique to the anterior, inferior, and external edge of the liver in $97 \%$ of cases and horizontal in $3 \%[5]$.

The orientation of RS as oblique or horizontal seen during laparoscopic cholecystectomy may change with orientation of camera and hence can be interpreted differently by different operating surgeons. Hence, this could be subject to bias.

6.4. Operative Time and RS. The operative time in RS visible group was reduced by around $25 \%$ in comparison to RS not visible group. In RS visible group, no time would have been spent in adhesion release, and the operating surgeons might 
TABLE 2: Intra/postoperative complications and RS.

\begin{tabular}{|c|c|c|c|}
\hline \multirow{2}{*}{ Complications } & \multicolumn{2}{|c|}{$\begin{array}{l}\text { RS visible before dividing cystic artery/duct } \\
\text { dissection group, } n=208\end{array}$} & \multirow{2}{*}{$\begin{array}{l}\text { RS not visible before dividing cystic artery/duct, } \\
\qquad n=22 \text { (absence of RS) }\end{array}$} \\
\hline & $\begin{array}{l}\text { RS visible initially, } \\
\qquad n=162\end{array}$ & $\begin{array}{l}\text { RS visible on release of } \\
\text { adhesion, } n=46\end{array}$ & \\
\hline $\begin{array}{l}\text { Major } \\
\text { (i) Common bile duct injury }\end{array}$ & 0 & 0 & 1 \\
\hline $\begin{array}{l}\text { Minor } \\
\text { (i) Port site SSI } \\
\text { (ii) Port site hematoma } \\
\text { (iii) Serosanguineous collection in } \\
\text { GB fossa }\end{array}$ & $\begin{array}{l}0 \\
1 \\
0\end{array}$ & $\begin{array}{l}0 \\
0 \\
1\end{array}$ & $\begin{array}{l}1 \\
1 \\
1\end{array}$ \\
\hline Total & & 2 & 4 \\
\hline
\end{tabular}

not had any preoccupied hesitancy regarding chance of CBD injury as RS marks the plane of CBD.

6.5. Duration of Hospital Stay and RS. The duration of hospital stay in the RS visible was significantly less than that in RS not visible group. The shorter hospital stay in RS visible group could be attributed to lesser operative time, lesser dissection, and lesser need to place drain in this group. Majority of patients with drain had tendency toward going home only after removal of drain.

6.6. Intra/Postoperative Complications and RS. 1 minor complication occurred in each group: RS visible initially group and RS visible on adhesion release group. On the other hand, 3 minor complications occurred in RS not visible group. Only one major complication (bile duct injury) occurred in RS not visible group.

Even though this discrepancy on occurrence of complication in various groups was statistically not significant because of small sample size, it could be clinically significant and it can be concluded that recognition of RS before dividing cystic artery/duct during laparoscopic cholecystectomy avoids major complications.

Peti and Moser described a case where identification of the sulcus helped to prevent common bile duct injury [8]. Hugh et al. and Zubair et al. had shown minimal common bile duct injury during laparoscopic cholecystectomy by beginning the dissection ventral to the RS $[4,9]$.

The overall incidence of bile duct injury (BDI) in our study was 1 in 230 cases, which amounts to $0.4 \%$. Flum et al. reported that the incidence of bile duct injury increased from $0.2 \%$ in open cholecystectomy era to $0.5 \%$ after laparoscopic cholecystectomy [10].

\section{Summary}

The RS was present in $90.4 \%$ of the cases, and the open sulcus type was the commonest (54\%), followed by the scar type $(22.9 \%)$, the closed sulcus type $(12.5 \%)$, and the slit type (10.6\%), respectively. In $59.1 \%$ of the cases, it was oblique to the anterior, inferior, and external edge of the liver, and in $40.9 \%$ of the cases, it was transverse. The mean $\pm S D$ values for operative time and duration of hospital stay in the RS visible and the RS not visible groups were $29.16 \pm 8.736$ and $42.9 \pm 23.646$ minutes, and $1.26 \pm 0.440$ and $1.90 \pm 0.910$ days, respectively ( $p$ value $\leq 0.001)$. One minor complication occurred in each group: RS visible initially group and RS visible on adhesion release group. On the other hand, 3 minor complications occurred in RS not visible group. Only one major complication occurred in RS not visible group.

\section{Conclusion}

Rouviere's sulcus is present in majority of population in our part of the world, and open sulcus type is the commonest. Recognition of RS before dividing cystic artery/duct during laparoscopic cholecystectomy avoids major complications and is associated with short operative time as well as short duration of hospital stay.

8.1. Limitation. This study had few limitations like small sample size, short term follow-up, observational bias, and being single center-based study.

8.2. Recommendation. Identification of RS is a predictor of safe laparoscopic cholecystectomy.

\section{Data Availability}

The data used to support the findings of the study can be made available from the corresponding author upon request.

\section{Additional Points}

Highlights. Well established: Rouviere's sulcus is present in $70-80 \%$ of population and is an important anatomical landmark as the common bile duct lies below its level. Newly established: Rouviere's sulcus is present in $90 \%$ population in our part of world and open sulcus type is the commonest one. Recognition of RS before dividing cystic artery/duct during laparoscopic cholecystectomy avoids major complication and is associated with short operative time as well as short duration of hospital stay. 


\section{Ethical Approval}

This study was performed in accordance with the principle of the Declaration of Helsinki and was approved by the Institutional Review Board on 26 March 2019.

\section{Conflicts of Interest}

The authors declare that they have no conflicts of interest.

\section{References}

[1] C. Couinaud, "The paracaval segments of the liver," Journal of Hepato-Biliary-Pancreatic Surgery, vol. 1, no. 2, pp. 145-151, 1994.

[2] J. A. Reynaud, A. Brack, J. P. Grivet, and Y. Trudelle, "Interaction of phospholipid vesicles with basic amphiphilic polypeptides," Peptides 1990, vol. 172, no. 6, pp. 490-492, 1991.

[3] Gans, "Study of anatomy of the intrahepatic structures and its repercussions of hepatic surgery," Ph.D. thesis, University of Nijmegen, Nijmegen, Netherlands, 1995.

[4] T. B. Hugh, M. D. Kelly, and A. Mekisic, "Rouvière's sulcus: a useful landmark in laparoscopic cholecystectomy," British Journal of Surgery, vol. 84, no. 9, pp. 1253-1254, 1997.

[5] R. Dahmane, A. Morjane, and A. Starc, "Anatomy and surgical relevance of Rouviere's sulcus," Scientific World Journal, vol. 2013, Article ID 254287, 4 pages, 2013.

[6] S. Sachdeva, M. Ansari, A. Anees, Z. Khan, and N. Khalique, "Lifestyle and gallstone disease: scope for primary prevention," Indian Journal of Community Medicine, vol. 36, no. 4, pp. 263-267, 2011.

[7] R. L. Gupta, S. B. Sharma, S. P. Kumar et al., "Changing trends in gall bladder stone disease- an observation," Indian Journal of Medical Sciences, vol. 52, pp. 309-316, 1998.

[8] N. Peti and M. A. J. Moser, "Graphic reminder of Rouviere's sulcus: a useful landmark in cholecystectomy," ANZ Journal of Surgery, vol. 82, no. 5, pp. 367-368, 2012.

[9] M. Zubair, L. Habib, F. Memon et al., "Rouviere's sulcus: a guide to safe dissection and laparoscopic cholecystectomy," Pakistan Journal Of Surgery, vol. 22, no. 2, pp. 119-121, 2009.

[10] D. R. Flum, A. Cheadle, C. Prela, E. P. Dellinger, L. Chan et al., "Bile duct injury during cholecystectomy and survival in medicare beneficiaries," JAMA, vol. 290, no. 16, pp. 2168-2173, 2003. 